\title{
Position Statement of the Brazilian Society of Sports Medicine and Brazilian Society of Geriatrics and Gerontology: Physical Activity and Health in the Elderly
}

\author{
Antonio Claudio Lucas da Nóbrega, Elizabete Viana de Freitas, Marcos Aurélio Brazão de Oliveira, \\ Marcelo Bichels Leitão, José Kawazoe Lazzoli, Ricardo Munir Nahas, Cláudio Aparício Silva Baptista, \\ Félix Albuquerque Drummond, Luciano Rezende, Josbel Pereira, Maurílio Pinto, \\ Rosana Bento Radominski, Neiva Leite, Edilson Schwansee Thiele, Arnaldo José Hernandez, \\ Claudio Gil Soares de Araújo, José Antonio Caldas Teixeira, Tales de Carvalho, \\ Serafim Ferreira Borges and Eduardo Henrique De Rose
}

\section{INTRODUCTION}

This publication represents the joint and official position statement of the Brazilian Society of Sports Medicine and Brazilian Society of Geriatrics and Gerontology about physical activity and health in elderly subjects. The purpose of this document is to make public current concepts concerning this issue in a practical and objective way, aiming to increase physical activity recommendation given by health professionals who deal with old people. The reader interested in more detailed information on these issues is recommended to consult the references listed at the end of this document.

\section{RATIONALE}

Life expectancy of the modern man has been increasing in last years, mostly as a consequence of the discover of several new medications that allowed a better control and more efficient treatment of chronic-degenerative and infectious diseases and also secondary to development of more efficient and sophisticated diagnostic and surgical techniques. The natural consequence was the increase of

1. This document was approved in a joint meeting of Brazilian Society of Sports Medicine and Brazilian Society of Geriatrics and Gerontology at the Universidade do Esporte do Paraná (Curitiba, PR, Brazil) on November $5^{\text {th }} 1999$.

2. Sponsors of this document: Iridium ${ }^{\mathrm{TM}}$ Brasil, Laboratórios Roche (Tilatil $\left.^{\circledR}\right)$, Tensor ${ }^{\circledast}$, Unimed Curitiba and Universidade do Esporte do Paraná.

3. Originally published in Revista Brasileira de Medicina do Esporte 1999; 5(6): 207-11.

4. This document can be reproduced as long as it is clearly identified as a joint official position statement of the Brazilian Society of Sports Medicine and Brazilian Society of Geriatrics and Gerontology - 1999. human's life average that today is placed around 66 years (20 years more than in 1950). Currently it is estimated that for every ten individuals, one is above 60 years of age. In developing countries people who are above this age are considered as elderly by the World Health Organization.

Population aging is a worldwide phenomenon that can also be seen in Brazil. According to data of the IBGE (Brazilian Institute of Geography and Statistics), by the year 2030 Brazil will have the sixth greatest world population of aged people in absolute numbers. The diseases linked to aging lead to a dramatic increase of health costs besides severe social consequences and deep impact in the economy of the countries. The majority of evidences show that the best way to improve and promote health in the elderly is to prevent its common medical problems. These interventions must be aimed specially at the prevention of cardiovascular diseases (CVD), considered the main cause of death in older people. On the other hand, a sedentary lifestyle, incapacity and dependence are the greatest health adversities related to aging. The main causes of incapacity are chronic diseases, including cerebrovascular accident sequelae, bone fractures, rheumatic diseases and CVD, among others.

The National Center of Statistics for Health estimates that about $84 \%$ of people aged 65 or above are dependent on others to carry out their daily activities.

It is estimated that in 2020 there will be seen an increase from 84 to $167 \%$ on the amount of old people suffering of moderate or serious incapacity. However, the implementation of preventive strategies, like practicing physical activity (PA) regularly and developing rehabilitation programs, should promote functional capacity improvement and minimize or prevent the appearance of such incapacity. 


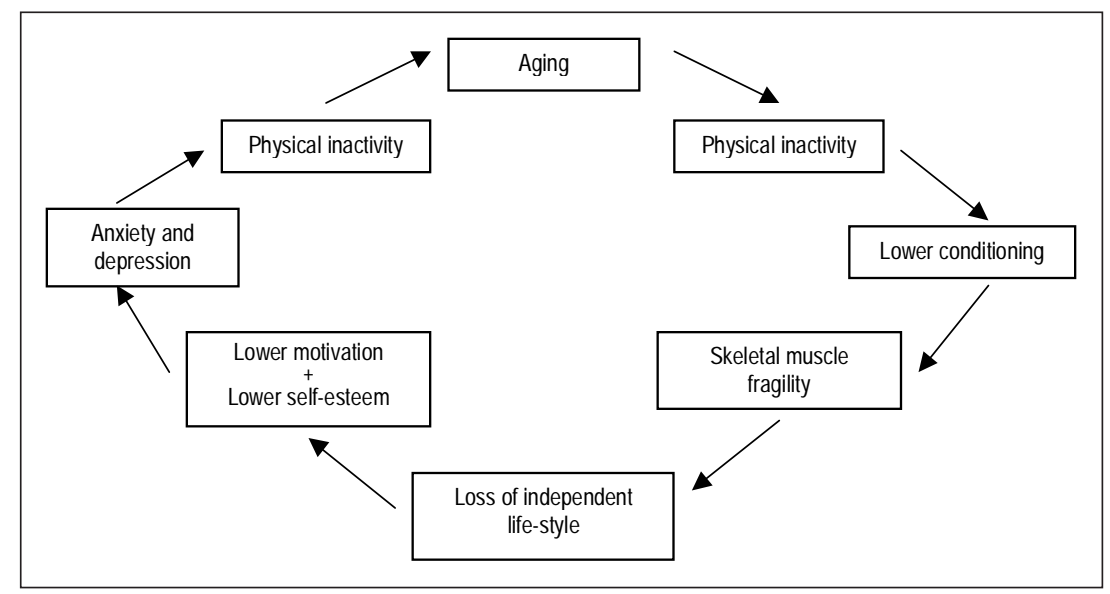

Fig. 1 - The vicious cycle of aging

\section{PHYSIOLOGY OF AGING}

Aging is a continuous process, during which occurs a progressive decline in all physiologic processes. Keeping an active and healthy lifestyle one should delay the morphologic and functional deterioration that occurs with age. Figure 1 shows schematically the vicious cycle of aging.

\section{CARDIOVASCULAR CHANGES OF AGING}

The increase in life expectancy improved the knowledge concerning physiological changes that occur in the cardiovascular system and skeletal muscle. However, it remains difficult to determine the narrow border between normal aging and pathological alterations.

Aging is associated with cardiac structural alterations that tend to be individualized. It is noticeable between the ages of 30 and 90 years an increase of cardiac muscle mass from 1 to $1.5 \mathrm{~g} /$ year. Left ventricular (LV) walls as well as the interventricular septum show a slight increase in thickness even in the absence of CVD, keeping normal echocardiographic indices. These alterations are related to a greater rigidity of the aorta, determining an increase in the impedance to the emptying of $\mathrm{LV}$, with a consequent increase of the afterload. Besides, there is collagen deposition, mainly in the LV posterior wall. Myocardial collagen infiltration increases heart rigidity. The systolic function remains unchanged, but there is a reduction of the LV compliance with impairment of the diastolic function, determining an increase in the time for ventricular relaxation. It is probable that those findings are related to the reduction of the reuptake of calcium by the sarcoplasmatic reticulum.

With aging, the autonomic nervous system modulation (adrenergic and vagal) of cardiac function diminishes, leading to a decline in the response to adrenergic stimulation on the senescent heart. The reduced beta-adrenergic re- sponse (lesser neural activation and reduction of beta-adrenergic receptors density) leads to a lower chronotropism, inotropism and arterial vasodilation. As a consequence, during exercise there is a reduction of the maximal heart rate (HR) and of maximal stroke volume (responsible for $50 \%$ of maximal oxygen consumption reduction consequent to aging).

The arteries suffer changes in their elasticity and compliance. The ventricular emptying in a less compliant aorta favors the increase of the systolic arterial pressure, while the increase of total peripheral vascular resistance determines a gradual increment of the mean arterial pressure. The aortic walls become thicker as a consequence of collagen and mucopolisacharids infiltration and calcium deposition, with loss of continuity of elastic layers. The pulse wave velocity is increased, reflecting vascular compliance reduction. The peripheral circulation suffers morphologic and functional alterations, such as the reduction of capillary/muscle fiber ratio, smaller capillary diameter and changes in the endothelial function. Specifically, it occurs a reduction in nitric oxide release and lower endothelial dependent vasodilator response, even though smooth muscle response to direct vasodilators remains unchanged.

These cardiovascular limitations lead to a reduction of maximum cardiac output that produces a reduction in the maximal oxygen consumption $\left(\dot{\mathrm{V}}_{2 \max }\right)$ of 0.4 to $0.5 \mathrm{ml}$. $\mathrm{kg}^{-1} \cdot \mathrm{min}^{-1} \cdot$ year ${ }^{-1}$ (i.e., $1 \%$ per year in the adult). Although genetic characteristics may influence the rate of $\mathrm{VO}_{2 \max }$ decline, the maintenance of regular physical activity can slow this reduction to $50 \%$.

\section{SKELETAL MUSCLE ALTERATIONS IN AGED PEOPLE}

In humans, neuromuscular system reaches its full maturation between 20 and 30 years of age. Between the third 
and forth decades maximal force remains unchanged or with no significant reductions. Around 60 years, a reduction of muscular maximal strength between 30 and $40 \%$ begins, that means a loss of strength of about $6 \%$ per decade from 35 to 50 years of age and of $10 \%$ per decade for individuals older than that.

A reduction of bone mass also occurs in older people, more frequently in women. When it occurs in higher levels this characterizes osteoporosis that can predispose to the occurrence of bone fractures.

After the age of 35 there are natural changes of articular cartilage that, associated with biomechanic alterations acquired or not, provokes several degenerations that can lead to locomotive function and flexibility reduction, carrying increased risk of injuries.

\section{EFFECTS OF PHYSICAL ACTIVITY IN AGED PEOPLE}

Regular physical activity is recommended to maintain and/or to improve the bone mineral density and to prevent bone mass loss. However, physical exercise must not be considered as a substitute of hormonal therapy. The association between drug treatment and physical activity is an excellent way to prevent bone fractures.

Physical activity improves muscular force and mass as well as articular flexibility, especially in individuals above 50 years of age. Old people's trainability (the capacity of physiological adaptation to exercise) does not differ from that of youngers.

Physical activity is an outstanding health device in all ages, especially in the elderly and brings several physiological and psychological adaptations, such as:

- increase of $\dot{\mathrm{V}}_{2 \max }$

- better peripheral vascular benefits

- increase of muscular mass

- better control of glycemia

- favorable changes in lipid profile

- reduction of body weight

- better control of resting blood pressure

- improvements of pulmonary function

- improving in balance and deambulation

- lower dependence for accomplishment of daily activities

- improvements of self-esteem and self-confidence

- significant improvement of the quality of life

Regular physical activity decreases the incidence of falls, risk of fractures and mortality in subjects with Parkinson's disease. For greater benefits, physical activity in those patients must include balance training exercises, walking and resistance exercises.
Physical activity has been indicated also for other neurological illnesses, such as multiple sclerosis and Alzheimer disease.

In aged people, regular physical activity, particularly weight-bearing and resistance exercises, promotes greater bone calcium fixation, assisting in prevention and treatment of osteoporosis. Physical activity also increases muscular strength and endurance, balance, and flexibility, consequently reducing fall incidence, bone fractures and its complications. Older people suffering of osteoarthrosis can also and must practice regular physical activity, as long as it is adapted to their condition.

\section{PRE-PARTICIPATION MEDICAL EVALUATION}

Although a pre-participation medical evaluation is a basic need, the unavailability must not hinder the adoption of an active life-style. Alternative evaluations go from simple questionnaires until sophisticated examinations. The main objectives of clinical examination are: identification of former and current illnesses, evaluation of nutritional status, medicine use, orthopedic limitations and current level of physical activity.

Amongst the complementary laboratory tests, the most important is the exercise test, whose main objectives are: determination of exercise tolerance and detection of exercise-induced myocardial ischemia. The basic reason for performing an exercise test in older people, even in asymptomatic ones lacking coronary disease risk factors, is that above 55 years of age the risk of coronary artery disease (CAD) exceeds $10 \%$, giving a great diagnostic value to this test, that is, a negative result reduces the risk of CAD to $2 \%$, while a positive result raises this risk to $90 \%$. The exercise test can include measurement of pulmonary ventilation and expired gases (ergospirometry or cardiopulmonary exercise test) that allows the direct measure of $\dot{\mathrm{VO}}_{2 \max }$, determination of ventilatory threshold and better identification of exercise intolerance.

The ideal pre-participation evaluation must still include muscular strength and flexibility tests, postural analysis and determination of body composition. The overall objective of these tests is to develop an individualized prescription, offering a higher improvement in quality of life when one is involved with recreational activities and optimizing performance when practice of a specific sport modality is intended.

\section{EXERCISE PRESCRIPTION}

When one considers exercise prescription for aged individuals, it must be considered - as in other ages - the different components of physical fitness: aerobic condition- 
ing, muscle endurance and strength, body composition and flexibility. The later assures mobility and agility maintenance, keeping independence and improving quality of life of aged people.

A physical activity program for aged groups must aim to break the vicious cycle of aging (Figure 1), improving aerobic conditioning and diminishing the deleterious effects of a sedentary life-style. It must also maximize social interaction, reducing anxiety and depression, common problems seen in older people.

As seen in other medical interventions, regular physical activity must follow some guidelines, in order to assure the best risk/benefit ratio. The main parameters to be observed for an adequate prescription are: modality, duration, frequency, intensity and mode of progression. However, it is important to emphasize that exercise planning must be individualized, using the pre-participation evaluation results and considering the co-morbidities.

Exercise modality choice must primarily take into account individual preferences and capabilities of aged people. After the pre-participation evaluation is completed, one or more modalities can be restricted in consequence of the diseases found in the individual. Leisure and socialization must be incorporated in a successful program. In order to accomplish this, the activities should, as frequent as possible, be performed in groups and be varied.

Some years ago, aerobic exercise prescription stated that they should be performed from 3 to 5 times a week, lasting 20 to 30 minutes, with intensity going from light to moderate. Alternatively to this formal prescription, a significant reduction of all-cause and cardiovascular mortality can be observed if one accumulates $2,000 \mathrm{kcal}$ or more of weekly energy expenditure. This energy expenditure can be reached through formal activities (for example: walking, swimming, hydrogimnastics) and also through daily and leisure activities, like stair climbing, accomplishing domestic tasks, gardening, dancing, etc.

More recent studies suggest that activities of intensity above 4.5 metabolic equivalents (METs) provide an additional reduction of the general and cardiovascular mortality of approximately $10 \%$. Thus, changing from an inactive state to light activities bring benefits. However, for those who already practice physical activity regularly, higher intensities are capable to increase even more those benefits.

However, in some aged individuals, their low functional capacity does not allow ideal exercise prescription. For those, an initial adaptation phase is required, in which intensity and duration will be determined in levels below those commonly used.

Physical activity must be initiated by a warm-up phase, range of motion exercises and stretching, along with the main activity at light intensities. Warm-up is an important phase as it reduces risk of injuries and increases blood flow to skeletal muscles. Progressive slow-down is equally important in preventing post-exercise hypotension. This complication can be more intense in the aged, as their hemodynamic adjustments are frequently slower and the use of cardiovascular acting drugs is common.

The intensity of aerobic phase can be determined using percentages of $\dot{\mathrm{VO}}_{2 \max }$ or of maximal HR measured directly in an exercise test or estimated by some equations. Cardiovascular acting drugs can modify the relationship between HR and exercise intensity. In this case, rating of perceived exertion scales can be used (Borg scale), an excellent alternative for any subject. Generally a moderate intensity is recommended, from 40 to $75 \%$ of $\dot{\mathrm{V}}_{2 \max }$ or from 55 to $85 \%$ of the HR maximal, what in general corresponds to 3 to 5 or 12 to 13 on Borg scales depending on which scale is used (0-10 or 6-20, respectively). It should be emphasized that high intensity sessions are associated with low rates of adherence secondary to muscular discomfort, especially at the initial phase.

The duration of activity can vary from 30 to 90 minutes, keeping an inverse relationship with the intensity. The so called "frail aged" and individuals in initial phases of an exercise program can benefit themselves from short duration sessions (five to ten minutes) carried out in two or more periods along the day.

Ideally, exercise should be performed in most of - if possible in all - the days of the week, in order to reach more easily the necessary energy expenditure to attain health benefits.

Safety is essential in the initial phase of an appropriate exercise program. Thus, educating practitioners about exercise principles and stimulating self-monitoring is important. Also, efforts should be done to make the exercise a habit as natural as, for example, taking care of one's personal hygiene.

For muscular strength and endurance training, large muscle groups should be involved. Two to three series of six to twelve bouts increase muscle strength and endurance. These series should be performed two to three times/ week, at an intensity equivalent to $60 \%$ of one maximal repetition.

Stretching exercises should be done avoiding ballistic movements. Progressive movements until a light discomfort is reached are adequate, and must take part of aerobic and strength sessions. In order to lessen the risk of injuries a higher level of care should be taken on movements' execution.

Safety is essential, not only from a cardiovascular perspective but also related to the locomotor system. It is im- 
portant to consider aged people's lower ability of adapting to temperature extremes and impaired control of hydration. Adequate information about clothes, footwear and hydration during the activity must be given. Also, it is important to assure well-illuminated and ventilated environments, and to avoid sliding surfaces.

Finally, physical activity programs must be stimulated for aged individuals, even by government or private institutions since they consist in an excellent health-promoting tool. These programs already exist in some Brazilian cities. No other segment of the population acquires more benefits with regular physical activity than the elderly.

\section{PRACTICAL RECOMMENDATIONS}

Some recommendations must be observed in order to assure a correct and safe exercise practice (Table 1):

\section{TABLE 1}

Recommendations to exercise practice

(1) Exercise only if there is a physical well-being.

7 Wear appropriate clothes and shoes.

$\checkmark$ Avoid smoking and taking sedatives.

$\checkmark$ Do not exercise after prolonged fasting. Prefer carbohydrates before exercise.

$\checkmark$ Respect individual limitation, suspending exercise if discomfort or pain occurs.

4 Avoid extremes of humidity and temperature.

$\checkmark$ Begin activities slowly and progressively to allow adaptation.

$\square$ Assure proper hydration before, during and after physical activities.

\section{CONCLUSIONS}

1. Regular physical activity improves quality of life and longevity of elderly people.

2. A physical activity program for aged people must be preceded by a medical evaluation and must also respect the different components of physical fitness, including aerobic, muscular strength, stretching and balance exercises.
3. Government at all levels, medical and scientific institutions, non-governmental organizations and the media must widespread the concept that physical activity is fundamental for health promotion of aged people and must also develop objective actions in order to make possible regular physical activity to this particular population.

\section{REFERENCES}

1. American College of Sports Medicine Position Stand on Osteoporosis and Exercise. Med Sci Sports Exerc 1995;27:i-vii.

2. American College of Sports Medicine. ACSM Position stand on exercise and physical activity for older adults. Med Sci Sports Exerc 1998; 30:992-1008

3. Blair SN, Kohl III HW, Paffenbarger Jr RS, Clark DG, Cooper KH, Gibbons LW. Physical fitness and all-cause mortality - A prospective study of healthy men and women. JAMA 1989;2395-401.

4. Carvalho T, Nóbrega ACL, Lazzoli JK, Magni JRT, Rezende L, Drummond FA, et al. Posição oficial da Sociedade Brasileira de Medicina do Esporte: atividade física e saúde. Rev Bras Med Esporte 1996;2: 79-81.

5. Fiatarone MA, Marks EC, Ryan ND, Meredith CN, Lipsitz LA, Evans WJ. High-intensity strength training in nonagenarians: effects on skeletal muscle. JAMA 1990;263:3029-34.

6. Fletcher GF, Balady G, Blair SN, Blumenthal J, Caspersen C, Chaitman B, et al. Statement on exercise: benefits and recommendations for physical activity programs for all Americans - A statement for health professionals by the Committee on Exercise and Cardiac Rehabilitation of the Council on Clinical Cardiology, American Heart Association. Circulation 1996;94:857-62.

7. Lee I-M, Hsieh C-C, Paffenbarger Jr RS. Exercise intensity and longevity in men - The Harvard Alumni Health Study. JAMA 1995;273: 1179-84.

8. Paffenbarger Jr RS, Hyde RT, Wing AL, Hsieh CC. Physical activity, all-cause mortality, and longevity of college alumni. N Engl J Med 1986;314:605-13.

9. Pate RR, Pratt M, Blair SN, Haskell WL, Macera CA, Bouchard C. Physical activity and public health - A recommendation from the Centers for Disease Control and Prevention and the American College of Sports Medicine. JAMA 1995;273:402-7.

10. Pollock ML, Mengelkoch L, Graves J, Lowenthal D, Limacher M, Foster C, et al. Twenty-year follow-up of aerobic power and body composition of older track athletes. J Appl Physiol 1997;82:1508-16.

11. Spirduso WW. Physical fitness, aging and psychomotor speed: a review. J Gerontol 1980;35:850-65.

12. WHO Expert Committee. Report of a rehabilitation after cardiovascular diseases, with special emphasis on developing countries. Geneva: Bulletin of the World Health Organization 1993. 